\title{
APENDICECTOMIA LAPAROSCÓPICA NA GESTANTE
}

\section{LAPAROSCOPIC APPENDECTOMY DURING PREGNANCY}

\author{
Fernando Antônio Santos Figueiredo, TCBC-RS ${ }^{1}$ \\ Carlos Otávio Corso, TCBC-RS ${ }^{2}$
}

\begin{abstract}
RESUMO: Objetivo: A apendicite aguda é a doença cirúrgica não-obstétrica mais comum da gravidez e pode ocorrer em qualquer idade gestacional. O seu diagnóstico freqüentemente é tardio, o que pode acarretar alto índice de perfuração e complicações. O presente estudo visa apresentar os resultados da videolaparoscopia no tratamento da apendicite aguda na gravidez. Método: São analisados retrospectivamente quatro casos de pacientes com suspeita de apendicite aguda avaliadas em serviço de cirurgia de urgência e submetidas à cirurgia videolaparoscópica no segundo trimestre da gestação. Resultados: A videolaparoscopia confirmou o diagnóstico de apendicite aguda em três casos e o outro tratava-se de cisto ovariano roto. Todas foram tratadas pelo método laparoscópico, sem necessidade de conversão, e receberam alta hospitalar em cerca de 72 horas. Não houve complicações pósoperatórias. As pacientes foram acompanhadas no restante da gestação, e os partos ocorreram na data prevista, sem complicações maternas ou para os recém-nascidos. Conclusão: A apendicectomia laparoscópica na gravidez revelou-se um procedimento seguro, que possibilita uma recuperação pós-operatória mais rápida e sem evidências de que interfira com o curso da gestação. Entretanto, estudos com maior número de casos são necessários para estabelecer o seu real valor.
\end{abstract}

Descritores: Apendicectomia videolaparoscópica; Apendicectomia na gravidez; Apendicite aguda; Gestação.

\section{INTRODUÇÃO}

O ciclo gravídico-puerperal provoca alterações no organismo materno, mas a gestante esta sujeita às mesmas doenças agudas que a mulher não grávida, com algumas características especiais ${ }^{1}$. A apendicite aguda na gravidez é a causa mais comum de doença abdominal não-obstétrica que determina a necessidade de cirurgia de urgência. $\mathrm{O}$ seu diagnóstico na grávida pode ser bastante difícil, mesmo para o cirurgião experiente. Os exames laboratoriais, de importância no diagnóstico de abdome agudo, sofrem alterações na gestação. A realização de exame radiológico convencional é contra-indicada durante a gravidez devido à possibilidade de indução de malformações fetais, sendo este risco maior no primeiro trimestre ${ }^{2}$. O emprego da ultra-sonografia para o diagnóstico de apendicite aguda apresenta taxas de sensibilidade e especificidade elevadas, principalmente no segundo e terceiro trimestres gestacionais ${ }^{3}$.
O retardo no diagnóstico leva a intervenções cirúrgicas tardias, constatando-se elevadas taxas de perfuração apendicular e peritonite, aumentando os índices de morbidade e mortalidade materna e fetal ${ }^{4}$. Assim, o diagnóstico e a cirurgia precoces são vitais para minimizar a ocorrência da perfuração. $\mathrm{O}$ aforisma de que "a mortalidade da apendicite na gestante é a mortalidade da demora"3 permanece ainda atual.

Recentemente a cirurgia videolaparoscópica tem sido realizada com sucesso na gestação, sendo a colecistectomia o procedimento mais executado ${ }^{2}$. Outros procedimentos relatados são a apendicectomia, cirurgias ovarianas e o tratamento cirúrgico de massa anexiais ${ }^{2}$. Os avanços obtidos com a cirurgia laparoscópica, como dor de menor intensidade, redução do tempo de hospitalização e retorno precoce às atividades regulares são vantagens integralmente transferidas às gestantes. A videolaparoscopia também pode trazer outros benefícios a essas pacientes, salientando-se o decréscimo da taxa de nascimentos pré-termo devido a me-

1. Cirurgião do Serviço de Cirurgia Geral do Hospital Mãe de Deus, Porto Alegre, Conselheiro da Sociedade de Cirurgia Videolaparoscópica - RS, Cirurgião do Centro Clínico Mãe de Deus.

2. Cirurgião do Serviço de Cirurgia Geral do Hospital Mãe de Deus, Porto Alegre, Doutor em Medicina, Prof. Adjunto do Departamento de Ciências Morfológicas e do Curso de Pós-graduação em Medicina:Cirurgia, UFRGS

Recebido em 19/10/2001

Aceito para publicação em 07/03/2002

Trabalho realizado no Serviço de Cirurgia Geral, Hospital Mãe de Deus, Porto Alegre, RS. 
nor manipulação do útero gravídico, menor depressão fetal secundária à diminuição do uso de narcóticos, redução na incidência de hérnia incisional e retorno precoce do peristaltismo intestinal. Entretanto, a possibilidade de acidose fetal devido à absorção de $\mathrm{CO}_{2}$, potencial diminuição do aporte sangüíneo arterial ao útero causado pelo aumento da pressão intra-abdominal e a hipotensão fetal são situações preocupantes, e permanecem em estudo ${ }^{5-7}$.

\section{MÉTODO}

Foi realizado estudo retrospectivo de quatro gestantes atendidas no Setor de Emergência do Hospital Mãe de Deus com suspeita de apendicite aguda no período compreendido entre 1996 e 2000. Todas as pacientes encontravam-se no segundo trimestre gestacional, sendo duas multíparas e duas primíparas com idades de 32 e 33 , e 21 e 25 anos, respectivamente. As pacientes multíparas foram submetidas a cesarianas nas gestações anteriores.

\section{RESULTADOS}

A dor intensa em abdome inferior com irritação peritoneal, hipertermia e leucocitose com desvio à esquerda foi o achado comum a todas as pacientes. $\mathrm{O}$ exame do sedimento urinário estava alterado em uma paciente, porém não caracterizava quadro de infecção. Não foi realizada investigação radiológica convencional. A ecografia foi empregada nas quatro pacientes e foi sugestiva de apendicite aguda em dois casos e inespecífica nos outros dois. Todas foram também avaliadas por obstetra, tendo sido descartadas complicações gestacionais.

As pacientes foram submetidas à anestesia geral endovenosa com o uso de diprivan. O esvaziamento prévio da bexiga dispensou o cateterismo vesical e foram posicionadas em decúbito dorsal com rotação lateral esquerda de 30 graus. O cirurgião e o auxiliar colocaram-se à esquerda da paciente. A agulha de Verres foi introduzida na região supra-umbilical após cuidadosa palpação do útero junto à parede abdominal e certeza do seu afastamento do local da punção. Em nenhum caso foi utilizada a técnica de Hasson. $\mathrm{O}$ pneumoperitônio foi realizado com a insuflação de $\mathrm{CO}_{2}$ de forma lenta e gradual, e a pressão na cavidade abdominal não ultrapassou os $12 \mathrm{mmHg}$ durante todo o ato operatório. A punção inicial foi feita com trocarte de $10 \mathrm{~mm}$. Os demais trocarteres foram introduzidos no hipocôndrio direito $(10 \mathrm{~mm})$ e fossa ilíaca esquerda $(5 \mathrm{~mm})$ sob visão direta. O diagnóstico pré-operatório de apendicite aguda foi comprovado em três casos, e o outro se tratava de um cisto hemorrágico roto de ovário direito.

Nos casos de apendicite aguda, o órgão foi tracionado pelo seu meso e dissecado, evitando-se manipulação excessiva. $\mathrm{O}$ meso foi exposto até a implantação do apêndice no ceco, a artéria apendicular foi isolada e duplamente clipada. Após ligadura tripla com fios de Endoloop ${ }^{\circledR}$ e secção junto à sua implantação no ceco, o apêndice foi removido da cavidade através do trocarte do hipocôndrio direito. A aponeurose nos portais de $10 \mathrm{~mm}$ foi aproximada com pon- tos de poliglactina e náilon na pele. $\mathrm{O}$ abdome foi irrigado com solução salina morna e o útero manipulado o mínimo possível. Não foram deixados drenos. Nenhum dos apêndices estava perfurado, e o exame anatomopatológico confirmou o diagnóstico de apendicite aguda supurativa em todos. Na paciente que apresentou cisto ovariano roto foi realizada ooforectomia. Não houve complicações maternas ou fetais no transcurso do ato cirúrgico/anestésico. A monitorização fetal não foi utilizada durante a cirurgia e também não foram empregados agentes tocolíticos profiláticos. Todas as pacientes receberam cefalotina durante 24 horas, com a dose inicial administrada na indução anestésica. O tempo cirúrgico médio foi de 60 minutos e a alta hospitalar em cerca de 72 horas. Uma paciente apresentou hipertermia no quinto PO. Foi reavaliada clínica e ecograficamente, não sendo detectada anormalidade relacionada com a cirurgia e/ou gravidez, com breve retorno à normalidade após tratamento sintomático.

Todas as gestantes apresentaram o restante do período de gravidez sem intercorrências, com gestações a termo. Em três foram realizadas cesarianas e a outra teve parto normal. Não houve nenhuma complicação materna, e os recém-nascidos apresentaram peso ideal, índice de Apgar acima de 8 no primeiro minuto, e 10 após o quinto minuto.

\section{DISCUSSÃO}

A cirurgia abdominal não-ginecológica de urgência é necessária em cerca de $0,2 \%$ das gestantes, sendo a apendicite aguda a causa mais freqüente, ocorrendo em aproximadamente 1:1.500 gestações ${ }^{2-4,6}$. Pode acontecer em qualquer idade da mulher, sendo mais comum abaixo dos 30 anos, o que não a diferencia de pacientes não-grávidas, e acomete qualquer período do ciclo gravidico ${ }^{1,2,4}$.

$O$ primeiro relato de apendicite aguda na gestante foi feito há mais de 150 anos, e até hoje seu diagnóstico é um desafio ao cirurgião, por mais experiente que seja, devido às alterações tanto de ordem fisiológica como anatômica que ocorrem na gestante ${ }^{3,8}$. Como consequiência do crescimento uterino, o apêndice pode ser deslocado para cima e para o lado direito, e no primeiro trimestre a dor se localiza na fossa ilíaca direita em $90 \%$ dos casos, $75 \%$ no segundo trimestre e apenas $37 \%$ no terceiro trimestre 9 .

A temperatura axilar raramente ultrapassa os $38^{\circ} \mathrm{C}$, desde que não haja perfuração ${ }^{8}$. Leucocitose discreta, dita fisiológica, é comum na gravidez, o que dificulta a interpretação do leucograma. Entretanto a contagem de granulócitos acima de $80 \%$ é significativa de processo inflamatório ou infeccioso ${ }^{8}$. A pielonefrite aguda é o diagnóstico diferencial mais comum de apendicite na gestação ${ }^{8}$.

O emprego da radiologia convencional para auxílio no diagnóstico é infrequiente, principalmente no primeiro trimestre, sendo o ultra-som recomendado como rotina na investigação do abdome agudo na gestante ${ }^{2,3}$.

Ainda que métodos de tratamento modernos tenham virtualmente eliminado a mortalidade materna nos últimos 25 anos, a mortalidade fetal ocorre em cerca de 1,5\%, e nos casos em que há perfuração apendicular pode chegar a $35 \%$. Como consequiência do crescimento uterino, o ceco é des- 
locado para cima e para o lado direito, e assim o epíploon teria maior dificuldade em bloquear o apêndice inflamado, o que poderia explicar a maior incidência de perfuração apendicular na gestante. A perfuração tem ocorrência duas a três vezes maior durante a gestação, apesar da gravidez por si só não ter influência, e é mais freqüente no terceiro trimestre $(69 \%)$, estando associada na maioria da vezes ao retardo da intervenção cirúrgica ${ }^{4,8}$. As elevadas taxas de laparotomias negativas em abdome agudo na gravidez, cerca de $35 \%$ a $50 \%$, são aceitáveis quando cotejadas com a tentativa de minimizar a morte fetal ${ }^{4,8}$. Pacientes gestantes com suspeita de apendicite aguda devem ser submetidas a exploração cirúrgica precoce, não obstante o trimestre gestacional em que se encontram.

$\mathrm{O}$ advento da vídeocirurgia revolucionou a cirurgia geral nos últimos anos, e as vantagens evidentes da laparoscopia também podem ser aplicadas à pacientes gestantes. Apesar de poucos cirurgiões contra-indicarem de maneira absoluta o método, outros o empregam com cautela devido aos efeitos ainda pouco conhecidos do $\mathrm{CO}_{2}$ sobre o feto ${ }^{6,7,9}$. A abordagem videolaparoscópica poderia reduzir o risco de irritabilidade uterina, aborto espontâneo ou trabalho de parto precoce, devido ao menor trauma cirúrgico ${ }^{9}$. Estudos realizados em animais não ofereceram conclusões definitivas, todavia alguns autores preconizam a videolaparoscopia como a abordagem de escolha para a colecistectomia na gestante ${ }^{5,9}$. Duncan et al. realizaram um estudo com 2.565 mulheres, com grupo controle, e concluíram não haver aumento do risco de anomalias congênitas entre os dois grupos ${ }^{10}$. Mazze e Kallen relataram pequena elevação na incidência de menor peso ao nascimento, e um aumento significativo de mortes de recém-nascidos até sete dias após o parto, em relação à cirurgia convencional ${ }^{11,12}$.

$\mathrm{O}$ feto normalmente apresenta discreta acidose respiratória. As trocas de $\mathrm{CO}_{2}$ através da placenta permitem que o excesso do gás seja eliminado na respiração materna. Se o $\mathrm{CO}_{2}$ materno encontra-se elevado, como durante o pneumoperitônio, estas trocas são prejudicadas e pode resultar em piora da acidose fetal. Enquanto mínima acidose é normal e benéfica, os efeitos de acidose moderada por uma ou duas horas durante a cirurgia laparoscópica são desconhecidos ${ }^{7}$.
É bem estabelecido que o aumento da pressão intraabdominal, associado ao pneumoperitônio, pode levar à diminuição do retorno venoso. O feto é extremamente sensível à estabilidade cardiopulmonar materna, e a causa primária de morte fetal são a hipotensão e/ou hipoxia ${ }^{6}$. Claramente, uma alteração do trabalho cardíaco materno pode levar ao sofrimento fetal. Também um aumento da pressão intra-abdominal pode diminuir o fluxo sangüíneo ao útero, ou aumentar a pressão intra-uterina, ambos levando à hipoxia fetal $^{6}$. Na tentativa de não prejudicar o retorno venoso da veia cava inferior, a gestante deve ser colocada em decúbito dorsal com rotação de 30 graus à esquerda para a realização da cirurgia. A monitorização fetal transoperatória deve ser empregada quando há sinais de sofrimento do feto, porém o emprego de drogas para prevenir o tromboembolismo e agentes tocolíticos é controverso ${ }^{6}$.

Acidentes devido à introdução da agulha de Verres no útero gravídico foram relatados ${ }^{5}$. Assim, a técnica de Hasson para a colocação dos trocarteres a céu aberto parece ser mais segura ${ }^{5}$. Todavia, em nossa experiência não houve necessidade, possivelmente pelo fato de todas as pacientes estarem no segundo trimestre, apresentando úteros de volume relativamente reduzidos.

A pressão da cavidade abdominal deve ser mantida o mais baixo possível, o suficiente para boa visualização visceral, elevando-se de maneira gradual e não deve ser maior que $12 \mathrm{mmHg}$.

O emprego de grampeadores mecânicos, eletrocautério ou fios para a ligadura apendicular são critérios do cirurgião. A identificação das estruturas inflamadas pode ser facilitada com a aplicação de cilindros de gaze para dissecção romba e hemostasia por compressão, mantendo o campo operatório limpo ${ }^{13}$. O emprego da laparoscopia também pode reduzir a taxa de apendicectomias desnecessárias $^{14}$.

Nossos resultados permitem concluir que a videolaparoscopia pode ser empregada com segurança na apendicectomia na gestante durante o segundo trimestre da gestação, conferindo os mesmos benefícios obtidos em outras pacientes. Entretanto, um maior número de casos e a avaliação do método no terceiro trimestre são necessários para estabelecer sua efetividade.

\begin{abstract}
Background: Acute appendicitis is the main abdominal non-obstetric surgical pathology during pregnancy. It may occur at any maternal age and in any gestational period. It is frequently diagnosed lately, leading to a high index of perforation and complications. The aim of the study was to evaluate the feasibility of videolaparoscopic appendectomy. Method: Four pregnant patients, all in the second trimester, who were attended at the Emergency Unit at Hospital Mãe de Deus with clinical suspicion of acute appendicitis, were submitted to videolaparoscopic exploration and were retrospectively evaluated. Results: Laparoscopy confirmed acute appendicitis in 3 cases and the other was a ruptured hemorrhagic ovarian cyst. All patients were treated laparoscopicaly, and no conversion was needed. There were no post-operative complications and all four were discharged within 48 hours. The patients were followed and the deliveries were carried out in a proper form, without maternal or newborn complications. Conclusions: In this study, laparoscopic appendectomy was a safe procedure. It allowed a fast recovery and there were no apparent interference with gestacional course. However more studies with a great number of cases are necessary, in order to establish its real value.
\end{abstract}

Key Words: Appendectomy; Videolaparoscopy; Pregnancy; Appendectomy during pregnancy. 


\section{REFERÊNCIAS}

1. Rasslan S, Orsi PR. Urgência abdominal não obstétrica na gestação. In: Rasslan S (ed). Afecções cirúrgicas de urgência. $2^{\mathrm{a}}$ edição. Rio de Janeiro. Robe Editora, 1995, pp 347-361.

2. Firstenberg MS, Malangoni MA. Gastrointestinal surgery during pregnancy. Gastro Clin N Am.1998, 27(1): 7389.

3. To WW, Ngai CS, Ma HK. Pregnancies complicated by acute appendicitis. Aust N Z Surg 1995, 65:799-803.

4. Al-Mulhim AA. Acute appendicitis in pregnancy. A review of 52 cases. Int Surg 1996, 81: 295-297.

5. Reedy MB, Galan HL, Richards WE et al. Laparoscopy during pregnancy. A survey of laparoendoscopic surgeons. J Reprod Med 1997, 42: 33-38.

6. Curet MJ, Allen D, Josloff RK et al. Laparoscopy during pregnancy. Arch Surg 1996,131: 546-551.

7. Amos JD, Schorr JS, Norman PF et al. Laparoscopic surgery during pregnancy. Am J Surg 1996, 171: 436437.

8. Sharp HT. Gastrointestinal surgical conditions during pregnancy. Clin Obst Gynecol 1994, 37(2): 306-315.

9. Curet MJ, Vogt DA, Schob O et al. Effects of $\mathrm{CO}_{2}$ pneumoperitoneum in pregnant ewes. J Surg Res 1996, 63: 339-344.

10. Duncan PG, Pope WB, Cohen MM et al. Fetal risk of anesthesia and surgery during pregnancy. Anesthesiology. 1986, 64: 790-794.

11. Mazze RI, Kallén B. Reproductive outcome following anesthesia and operation during pregnancy.: a registry study of 5405 cases. Am J Obstet Gynecol 1989, 161: 1178-1185.
12. Mazze RI, Källén B. Appendicectomy during pregnancy: a Swedish registry study of 778 cases. Obstet Gynecol 1991, 77: 835-840.

13. Miguel PR, Figueiredo FA, Rosa ALM. Contribuição técnica para a colecistectomia laparoscópica: utilização do cilindro de gaze. Rev Col Bras Cir 1993, 20: 42-43.

14. Cox MR, McCall J, Padbury RT. Laparoscopic surgery in women with a clinical diagnosis of acute appendicitis. Med J of Aust 1995,162:130-132.

Endereço para correspondência:

Dr. Fernando Antônio Santos Figueiredo

Centro Clínico Mãe de Deus

Rua Costa, 30 conj. 505,

90110-270 — Porto Alegre-RS 